\title{
An EGARCH-BPNN system for estimating and predicting stock market volatility in Morocco and Saudi Arabia: The effect of trading volume
}

\author{
Salim Lahmiri*
}

Department of Computer Science, University of Quebec at Montreal, Montreal, Canada

\begin{tabular}{|c|c|}
\hline A R T I C L E I N F O & A B S T RAC T \\
\hline $\begin{array}{l}\text { Article history: } \\
\text { Received October 1, } 2011 \\
\text { Received in Revised form } \\
\text { November, 14, } 2011 \\
\text { Accepted } 30 \text { January } 2012 \\
\text { Available online } \\
20 \text { February } 2012 \\
\text { Keywords: } \\
\text { EGARCH } \\
\text { Volatility Forecasting }\end{array}$ & $\begin{array}{l}\text { In this study, the backpropagation neural network (BPNN) is tested for the ability to forecast } \\
\text { the daily volatility of two stock market indices from the Middle East and North Africa (MENA) } \\
\text { region using volume; namely Morocco and Saudi Arabia. Volatility series were estimated using } \\
\text { the Exponential Auto-Regressive Conditional Heteroskedasticity (EGARCH) model. The } \\
\text { simulation results show that trading volume helps improving the forecasting accuracy of BPNN } \\
\text { in Morocco but not in Saudi Arabia. As a result, volume represents valuable information flow } \\
\text { to be used in the modeling and prediction of volatility in Morocco. In addition, it is found that } \\
\text { BPNN overpredicts volatility during high volatile periods. This finding is important in financial } \\
\text { applications such as asset allocation and derivatives pricing. }\end{array}$ \\
\hline
\end{tabular}

\section{Introduction}

Volatility plays a central role in derivatives pricing, optimal portfolio selection, value-at-risk calculations used in portfolio risk management, and decision making in real-time trading systems. As a result, an extensive literature on volatility forecasting is motivated by these applications. Indeed, a number of statistical techniques have been developed to model and predict stock returns, such as GARCH family and stochastic volatility models. Besides, a strong link between contemporaneous trading volume and conditional volatility was found in the literature (Biswas \& Gerard, 2007). Then, one can use volume to forecast future volatility. This relationship was documented since early 1970s. For instance, Clark (1973) introduced the Mixture of Distribution Hypothesis (MDH) to document the contemporaneous relationship between stock returns and volume. Clark (1973) and Epps and Epps (1976) suggested that the information arrival in the equity market can be represented by volume. In other words, volume is a proxy for the information flow. Since then, the MDH has received a large attention in the academic literature. For example, Lamoureux and Lastrapes (1990) found evidence that trading volume explains the variance of US stock returns. Furthermore, they documented a substantial reduction in volatility persistence when volume is included in the variance

\footnotetext{
* Corresponding author. Tel: +1-514-649-7771.

E-mail addresses: lahmiri.salim@courrier.uqam.ca (S. Lahmiri) 
equation. Similarly, Omran and McKenzie (2000) reported that trading volume explains the variance of UK stock returns and the estimates of the GARCH process are insignificant when volume is included in the conditional variance equation.

In a subsequent study, Huang and Yang (2001) concluded that the volatility persistence of Taiwanese stock market declines significantly when volume is introduced as explanatory variable. Thus, they reject the MDH in the Taiwanese equity market. Lee and Rui (2002) found a positive relationship between volume and volatility in the US, UK and Japan stock market. In another study, Bohl and Henk (2003) concluded that the inclusion of trading volume in the conditional variance reduces volatility persistence in most of the Polish stocks. Therefore, they concluded that the MDH implications are not confirmed. Brooks (1998) concluded that augmenting models of volatility with measures of lagged volume leads only to very modest improvements in forecasting performance. Hu and Tsoukalas (1999) examined the individual volatility forecasts produced by a number of GARCH models in the European Monetary System (EMS). They concluded that the neural network combining model performed better in the crisis period and it was proved superior to linear combining models. Tseng et al., (2009) examined the variance forecasting ability of the traditional GARCH model and Grey-GARCH model using international stock indices. They found that the integrated Grey-GARCH model provides a better measure of the value of the forecasts than the simple GARCH model. They concluded that the integrated model help enhance the one-period-ahead volatility forecasts.

Other studies used artificial intelligence techniques to predict future stock market volatility because; unlike statistical models; they are nonlinear models that are capable to model noisy data. For instance, Hamid and Iqbal (2004) used artificial neural networks (ANN) for forecasting the volatility of the S\&P 500 Index futures prices. They found that forecasts from neural networks outperformed implied volatility forecasts and that those forecasts were not significantly different from realized volatility. Roh (2007) found that NN-EGARCH showed a good performance when compared with the NN and NN-GARCH model to forecast the KOPSI volatility. Bildirici and Ersin (2009) combined ARCH/GARCH family models with artificial neural networks to predict daily volatility of Istanbul Stock Exchange. Wang et al., (2011) used backpropagation neural network to predict TXO (Taiwan exchange options and futures) price under different volatility models, including historical volatility, implied volatility, deterministic volatility function, GARCH and Grey model combined with GARCH. They concluded that in general the prediction accuracy depends on volatility models and number of neurons in the hidden layer, but are not significantly related to activation functions. Hung (2011) used a fuzzy system to analyze clustering in generalized autoregressive conditional heteroskedasticity (GARCH) models and genetic algorithms to estimate the parameters of the membership functions and the GARCH models. Using data from developed market (Germany, Canada, Japan, and USA) the simulations showed that the proposed method improved the forecasting accuracy in comparison with conventional GARCH and EGARCH model. More recently, Hajizadeh et al. (2012) proposed a hybrid system that combines exponential GARCH and artificial neural networks to forecast the volatility of S\&P 500 index 10-days and 15-days ahead. The simulations indicated that the proposed system outperforms the conventional exponential GARCH model alone.

The main goal of this study is to forecast stock market future volatility using volume in two emergent markets from the Middle East and North Africa (MENA) region, while most of previous studies focused on developed and Asian markets (Omran \& McKenzie, 2000; Huang \& Yang, 2001; Lee \& Rui, 2002; Bohl \& Henk, 2003; Tseng et al., 2009). Two important stock markets in MENA region are considered: Morocco and Saudi Arabia. They are the most active market in the Middle East and North Africa respectively. The EGARCH model is used to estimate and extract stock market volatility. Then, backpropagation neural network (BPNN) is employed to model the estimated volatility and perform forecasts since they are robust to noisy data and capable to model nonlinear relationships (Rumelhart et al., 1986; Atsalakis \& Valavanis, 2009). Indeed, volatility and volume series are nonlinear and it is appropriate to approximate their relationship using nonlinear intelligent techniques such as artificial neural networks. In addition, BPNN has proven its capability to 
outperform traditional GARCH family models in the prediction of volatility (Hamid \& Iqbal, 2004, Roh, 2007, Bildirici \& Ersin, 2009; Hung, 2011, Wang et al., 2011, Hajizadeh, 2012).

The reminder of the paper is organized as follows: Section 2 outlines the data and volatility estimation. Section 3 describes the forecasting technique; namely the artificial neural networks. Section 4 reports the results. Finally, section 5 concludes.

\section{Data and volatility estimation}

The sample consists of daily index closing prices and trading volume. The sample covers observations for the period January 2000 to June 2006 for Morocco and Saudi Arabia. The series are taken from their respective stock market official websites. The daily return data are the first difference of the $\log$ of stock prices. i.e.. $R_{t}=100 *\left(\log P_{t}-\log P_{t-1}\right)$ where $P$ is the index price at time $t$. All returns are in local currency. Similarly, the change in volume at time $t$ is defined by the variable $V_{t}=100 *\left(\log v_{t}-\log v_{t-1}\right)$; where $v$ is the trading volume at time $t$.

To estimate volatility series, a two-step process is followed. First, the class of autoregressive moving average (ARMA) models (Davidson \& MacKinnon, 2008) is used for conditioning on the past of stock market return series to model the return processes (see equation 1). This class allows capturing all linear dynamics in return series. Second, the Exponential GARCH (EGARCH) model (Nelson, 1991) is used to estimate stock returns volatility (see equation 3). It is an extension of the GARCH model of Bollerslev (1986) to account for asymmetric volatility.

The EGARCH model is estimated using the maximum likelihood method (Davidson \& MacKinnon, 2008). Hence, the following model is estimated for each market index:

$$
R_{t}=C+\sum_{i=1}^{p} a_{i} R_{t-i}+\sum_{j=1}^{q} b_{j} \varepsilon_{t-j}+\varepsilon_{t} \text {, }
$$

where,

$\varepsilon_{\mathrm{t}} \sim \mathrm{GED}^{1}\left(0, h_{t}\right)$

and,

$\log \left(h_{t}\right)=w+\sum_{i=1}^{m} \Phi_{j}\left[\frac{\left|\varepsilon_{t-i}\right|}{\sqrt{h_{t-i}}}-\sqrt{2 / \pi}\right]+\gamma \frac{\varepsilon_{t-1}}{\sqrt{h_{t-i}}}+\sum_{j=1}^{n} \beta_{j} \log h_{t-j}+\eta_{t}$,

where $w, \Phi_{j}, \gamma, \beta_{j}$ and $\omega$ are constant parameters.

The EGARCH model imposes no restrictions on these parameters. The variance component $h_{t}$ is defined as an asymmetric function of lagged disturbances $\varepsilon_{t-i .}$. Hence, unexpected return variance $\varepsilon_{t}$ is modeled as an $\operatorname{EGARCH}(m, n)$. The selection of the $\operatorname{ARMA}(p, q)$ and $\operatorname{EGARCH}(m, n)$ processes are based on the minimization of Akaike and Schwarz information criterions (Davidson \& MacKinnon, 2008). Then, the conditional variance series - say volatility series given by $\log \left(h_{t}\right)$ - are extracted. Figure 1 shows volatility and changes in volume series for each market. The latter are computed as log volume first difference series as mentioned before. Finally, the estimated conditional variance series from the EGARCH model and volume are normalized in the interval $[-1,1]$ to obtain accurate forecasts. Then, normalized data will be fed to the backpropagation neural networks (BPNN). The normalization is performed as follows:

$$
x^{\prime}=\frac{(2 * x-(\operatorname{Max}[x]+\operatorname{Min}[x]))}{\operatorname{Max}[x]-\operatorname{Min}[x]},
$$

\footnotetext{
${ }^{1}$ Generalized Error Distribution (GED) was introduced by Subbotin (1923). Nelson (1991) used the GED to model the distribution of stock market returns.
} 
where $x$ and $x^{\prime}$ are respectively the original and the transformed data.
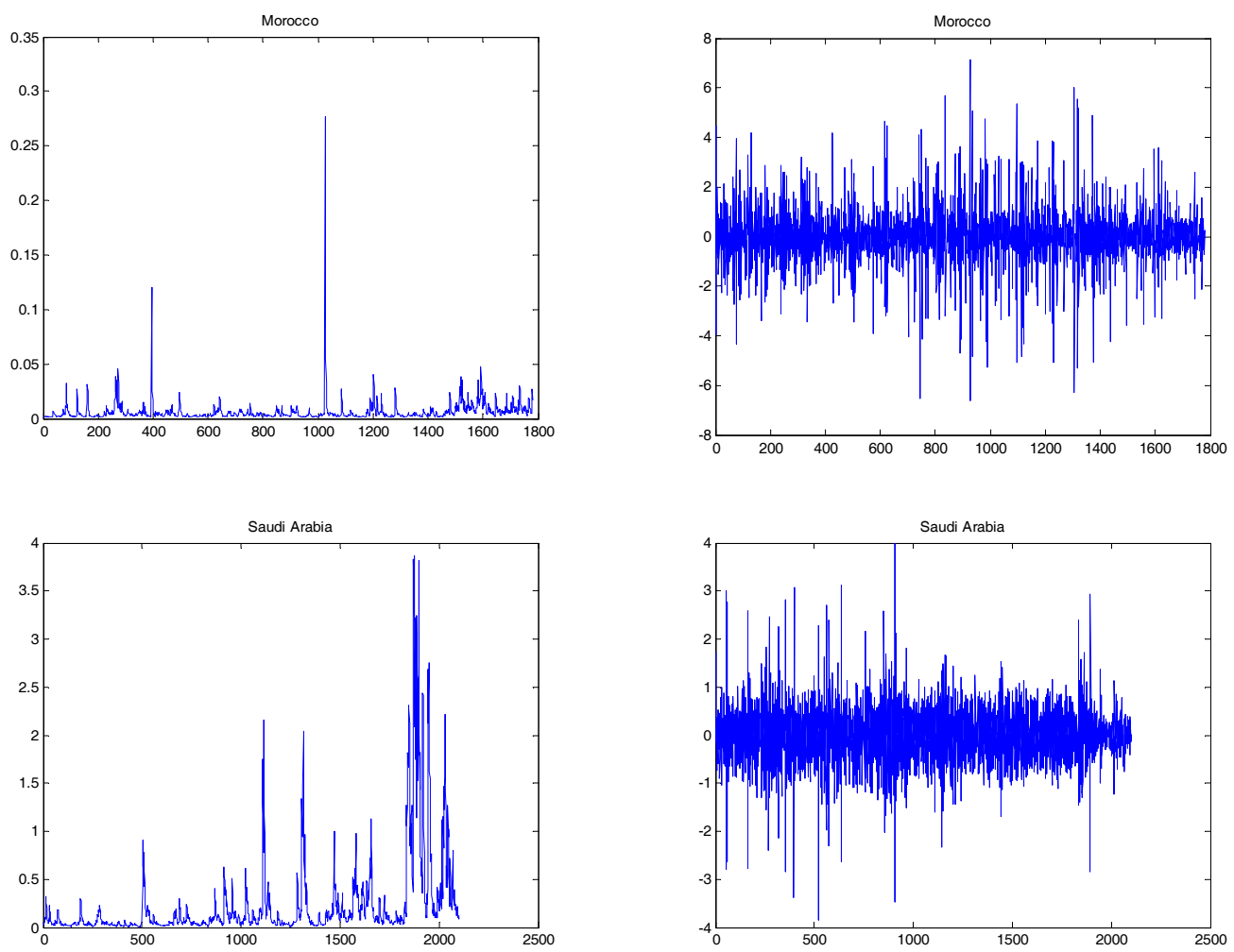

Fig. 1. Volatility (left column) and changes in volume series (right column).

\section{Artificial neural networks}

The main objective of this work is to predict the daily stock market volatility using backpropagation neural network (BPNN) (Rumelhart et al., 1986). The input data are the actual market volatility $\log \left(h_{t}\right)$ and the actual change in market volume $V_{t}$, and the output is the future market volatility $\log \left(h_{t+1}\right)$. The topology of the network is as follows: two neurons in the first layer, 4 neurons in the hidden layer, and one neuron in the third layer. The sigmoid transfer function - for example $f(x)=$ $1 /\left(1+e^{-x}\right)$ - is used in the hidden layer and a linear transfer function in the output layer. The final output has the form:

$O_{k}=f\left(\sum_{j=1}^{s} f\left(\sum_{i=1}^{n} x_{i} w_{i j}\right) w_{j k}\right)$,

where $n$ is number of input units, $s$ is number of neurons in hidden layer, and $m$ number of output neurons. And, $w_{i j}$ denotes the connection strength between units $i$ and $j$, and $k$ denotes the number of output units. The objective is to minimize the quadratic cost function $E$ which is computed as follows:

$E=\frac{1}{2} \sum_{\gamma=1}^{p} \sum_{k=1}^{m}\left(O_{k}^{\gamma}-y_{k}^{\gamma}\right)^{2}$,

where $p$ is number of patterns in the training set, and $O$ and $y$ are the actual and the desired output respectively. The solution of equation 6 is found using the backpropagation learning algorithm that updates the network weights and biases in the direction in which the performance function decreases most rapidly: the negative of the gradient. In our study, the network is trained with the LevenbergMarquardt (L-M) numerical algorithm (Nocedal \& Wright, 2000) where the weights are adjusted as follows: 
$\Delta w=w_{k+1}-w_{k}=-\left[J^{T} J+\mu I\right]^{-1} J^{T} e$,

where $J$ is the Jacobian matrix (first derivatives), $e$ is a vector of network errors (Eq. 6), and $\mu$ is a given parameter. The L-M algorithm approximates the Hessian matrix by computing the Jacobian matrix which is less complex than directly computing the Hessian matrix. As a result, the L-M numerical algorithm is fast. The parameter $\mu$ is arbitrarily set to 0.001 in our study.

Finally, in order to check the effectiveness of volume series in the prediction of future volatility, two models are simulated. The first one includes past volatility and past volume changes to predict future volatility. For instance, the following model is approximated and simulated:

$\log \left(h_{t+1}\right)=\Psi\left(\log \left(h_{t}\right), V_{t}\right)$,

where $\log \left(h_{t}\right)$ and $V_{t}$ are respectively the estimated volatility and changes in log volume series as defined in previous section. And, $\psi$ is the unknown nonlinear function to be approximated with artificial neural networks. The second one includes only past volatility to predict future volatility. For instance, the following model is approximated and simulated:

$\log \left(h_{t+1}\right)=\Phi\left(V_{t}\right)$

where $\Phi$ is the unknown nonlinear function to be approximated with artificial neural networks.

\section{Results}

Training and test sets were chosen as follows: first $80 \%$ observations were used as the training set and next $20 \%$ observations were used as the test set. The prediction performance is evaluated using the root mean of squared errors (RMSE), mean absolute error (MAE), and mean absolute deviation (MAD). They are defined as follows:

$$
\begin{aligned}
& R M S E=\sqrt{\frac{1}{N} \sum_{t=1}^{N}\left(E_{t}-P_{t}\right)^{2},} \\
& M A E=\frac{1}{N} \sum_{t=1}^{N}\left|E_{t}-P_{t}\right|, \\
& M A D=\frac{1}{N} \sum_{t=1}^{N}\left|P_{t}-\bar{P}\right|,
\end{aligned}
$$

where $E_{t}, P_{t}$, and $\bar{P}$ are respectively the estimated volatility, predicted volatility and the average of predicted volatility over the testing (out-of-sample) period; for example $t=1$ to $N$. Table 1 shows the details of the results obtained for each market. As shown in Table 1, volume series improve the prediction accuracy in the case of the Moroccan stock market. For instance, the obtained RMSE, MAD, and MAE when volume is included (excluded) as predictive variable are respectively 0.0062 (0.0113), 0.0036 (0.0055), and 0.0035 (0.0083). This finding is consistent with Lamoureux and Lastrapes (1990) and with Omran and McKenzie (2000) where GARCH models were used to estimate and predict future volatility. In addition, the obtained simulation results for Morocco indicate that volume decreases the prediction errors by $0.0051,0.0019$, and 0.0048 basis points for RMSE, MAD, and MAE respectively. In other words, volume modestly improves the forecasting accuracy of the BPNN. This finding is consistent with Brooks (1998) who used GARCH process to estimate and predict future volatility.

Besides, as shown in Table 1 volume series do not improve the prediction accuracy in the case of the Saudi Arabian stock market. The obtained RMSE, MAD, and MAE when volume is included 
(excluded) as predictive variable are respectively 0.3625 (0.3294), $0.1803(0.1643)$, and 0.1693 (0.1519). Thus, volume increases the prediction errors by $0.0331,0.016$, and 0.0174 basis points for RMSE, MAD, and MAE respectively. The finding is consistent with Huang and Yang (2001) and Bohl and Henk (2003) where GARCH models were used to estimate and predict future volatility.

Fig. 2 compares the estimated and the predicted volatility for each market. It clearly indicates that predicted volatility is higher than the estimated volatility during volatile periods for both markets and whether volume is considered as predictive variable or not. This finding is more pronounced in the case of the Moroccan stock market.

In sum, the simulations results indicate that trading volume helps improving the forecasting accuracy of BPNN in the prediction of stock market volatility in Morocco but not in Saudi Arabia. In other words, trading volume contains valuable information to be used to predict future volatility in Morocco. However, the obtained results suggest that volume contains noisy information that reduces the forecasting accuracy in Saudi Arabia.

Table 1

Performance measures

\begin{tabular}{lllll}
\hline & $\begin{array}{l}\text { Morocco } \\
\text { With Volume }\end{array}$ & $\begin{array}{l}\text { Morocco } \\
\text { No Volume }\end{array}$ & $\begin{array}{l}\text { Saudi Arabia } \\
\text { With Volume }\end{array}$ & $\begin{array}{l}\text { Saudi Arabia } \\
\text { No Volume }\end{array}$ \\
\hline RMSE & 0.0062 & 0.0113 & 0.3625 & 0.3294 \\
MAD & 0.0036 & 0.0055 & 0.1803 & 0.1643 \\
MAE & 0.0035 & 0.0083 & 0.1693 & 0.1519 \\
\hline
\end{tabular}
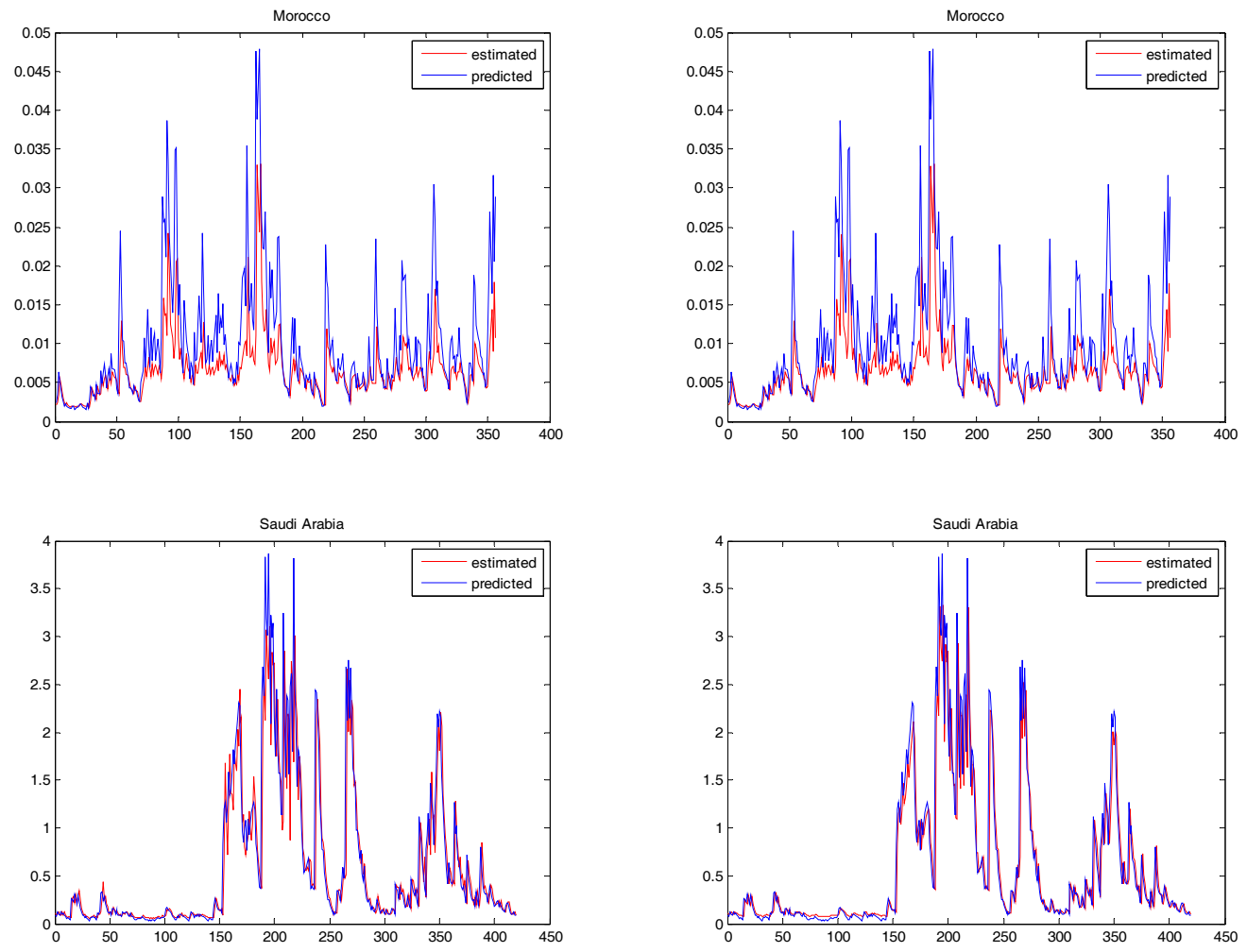

Fig. 2. Simulations with volume (left column) and when volume is excluded (right column) 


\section{Conclusion}

The predictability of market volatility is important for practitioners to allocate assets and determine expected portfolio return. Moreover, it is fundamental to forecast closing prices of derivatives such as options. In this study, artificial neural networks with backpropagation learning algorithm and Levenberg-Marquardt (L-M) numerical training algorithm were tested for their ability to forecast the stock market future volatility in Morocco and Saudi Arabia. EGARCH model was used to estimate volatility in each market. Then, neural networks were used to simulate forecasts. It is found that trading volume improves the forecasting accuracy of artificial neural networks in the prediction of stock market volatility in Morocco. Thus, volume contains valuable information to be used to predict future volatility in Morocco. However, the obtained results suggest that trading volume contains noisy information that reduces the forecasting accuracy in Saudi Arabia. In addition, it is found that the predicted volatility is larger than the estimated volatility during volatile periods for both markets. As a result, high level of predicted volatility should be taken with caution in practice; namely in portfolio management and derivatives evaluation. For future work, post 2008 financial crisis period will be considered along with other Middle East stock markets.

\section{References}

Atsalakis, G.S., \& Valavanis, K.P. (2009). Surveying stock market forecasting techniques - Part II: Soft computing methods. Expert Systems with Applications, 36 (3), 5932-5941.

Bildirici, M., \& Özgür, Ö.E. (2009). Improving forecasts of GARCH family models with the artificial neural networks: An application to the daily returns in Istanbul Stock Exchange. Expert Systems with Applications, 36, 7355-7362.

Bohl, M.T., \& Henke, H. (2003). Trading volume and stock market volatility: The Polish Case. International Review of Financial Analysis, 12, 513-525.

Bollerslev, T. (1986). Generalized Autoregressive Conditional Hetroscedasticity. Journal of Econometrics, 31, 307-327.

Brooks, C. (1998). Predicting stock index volatility: Can Market Volume help? Journal of Forecasting, 17, 59-98.

Clark, P.K. (1973). A subordinated stochastic process model with finite variance for speculative prices. Econometrica, 41, 135-156.

Davidson, R., \& Mackinnon, J.G. (2008). Econometric Theory and Methods. Oxford University Press, International Edition.

Epps, T. \& Epps, M. (1976). The stochastic dependence of stochastic price changes and transaction volume: Implications for the mixture of distribution hypothesis, Econometrica, 44, 305-321.

Girard, E., \& Biswas, R. (2007). Trading volume and market volatility: developed versus emerging stock markets. The Financial Review, 42, 429-459.

Hajizadeh E., Seifi, A., Zarandi Fazel M.H., \& Turksen, I.B. (2012). A hybrid modeling approach for forecasting the volatility of S\&P 500 index return. Expert Systems with Applications, 39, 431-436.

Hamid, S.A., \& Zahid, I. (2002). Using neural networks for forecasting volatility of S\&P 500 index futures prices. Journal of Business Research, 5881, 1-10.

Hu, M.Y., \& Tsoukalas, C. (1999). Combining conditional volatility forecasts using neural networks: An application to the EMS exchange rates. Journal of International Financial Markets, Institution and Money, 9, 407-422.

Huang, B.-N., \& Yang, C.-W. (2001). An empirical investigation of trading volume and return volatility of the Taiwan stock market. Global Finance Journal, 12, 55-77.

Hung, J.-C. (2011). Applying a combined fuzzy systems and GARCH model to adaptively forecast stock market volatility. Applied Soft Computing, 11, 3938-3945.

Lamoureux, C.G., \& Lastrapes, W.D. (1990). Heteroskedasticity in stock returns data: volume versus GARCH effects. The Journal of Finance, 45 (1), 221-229. 
Lee, B.-S., \& Rui, O.M. (2002). The dynamic of relationship between stock returns and trading volume: domestic and cross-country evidence. Journal of Banking and Finance, 26, 51-78.

McKenzie, E., \& Omran, M.F. (2000). Heteroskedasticity in stock returns data revisited: volume versus GARCH effects. Applied Financial Economics, 10, 553-560.

Nelson, D.B. (1991). Conditional heteroskedasticity in asset returns: a new approach. Econometrica, 59, 347-370.

Nocedal, J., \& Wright, S.J. (2000). Numerical optimization. Springer.

Roh, T.H. (2007). Forecasting the volatility of stock price index. Expert Systems with Applications, 33, 916-922.

Rumelhart, D.E., Hinton, G.E., \& Williams, R.J. (1986). Learning internal representations by error propagation. In Rumelhart, D.E. and J.L. McClelland, eds. Parallel distributed processing:explorations in the microstructure of cognition. Cambridge, MA, MIT Press, 318-362.

Subbotin, M.T. (1923). On the law of frequency error. Matematicheskii Sbornik, 31, 296-301.

Tseng, C.-H., Cheng, S.-T., \& Wang, Y.-H. (2009). New Hybrid Methodology for Stock Volatility Prediction. Expert Systems with Applications, 36 (2), 1833-1839.

Wang, C.P., Lin, S.H., Hung-Hsi Huang, H.H., \& Wu, P.C. (2011). Using neural network for forecasting TXO price under different volatility models. Expert Systems with Applications, 39(5), 5025-5032. 